\title{
Análisis de las concepciones del profesorado de Educación Infantil sobre la enseñanza de la lengua oral: un estudio de caso
}

\author{
Analysis of the conceptions of teachers at Infant \\ Education on the teaching of oral language: a cases study
}

María Santamarina Sancho*

Recibido: 29 de julio de 2011

Aprobado: 31 de octubre de 2011

\section{Resumen}

Se presentan resultados de una investigación empírica de carácter cualitativo. Concretamente, se trata de un estudio de casos que utiliza como instrumento y método de recolección de datos la entrevista clínica, con la que pretendemos acceder al conocimiento y a las concepciones que poseen los docentes de Educación Infantil sobre la enseñanza de la lengua oral en esta etapa educativa. Una vez recogida la información, se hizo su análisis y categorización utilizando como herramienta el software de análisis de datos cualitativos nvivo v.9. Con la investigación aquí iniciada se pretende, como consecuencia de las evidencias encontradas y expresadas en las conclusiones obtenidas, poner de manifiesto que hay una clara necesidad de mejorar y actualizar tanto la formación inicial como la formación permanente del profesorado de Educación Infantil, desde un punto de vista científico y didáctico, en lo relativo a la competencia oral y al modo de lograr su desarrollo en el alumnado de esta etapa.

Palabras clave: didáctica de la lengua oral, educación Infantil, concepciones del profesorado, formación del profesorado.

\section{Abstract}

This article presents the results of an empirical investigation of a qualitative nature, specifically this investigation is a cases study that we used as instrument and method of collection of data the clinical interview with which we intend to access knowledge and conceptions that they have got about the oral language in the Infant education. Once collected the information, we have made the analysis and categorization with the software Nvivo-v.9 (qualitative data analysis). With this research we want to show that it is very important to improve and update the training of teachers of Infant Education in order to increase students develop oral language in an appropriate manner.

Key words: didactics of oral language, infant education, conceptions, teacher education.

Magíster en Investigación e Innovación en Currículum y Formación. Departamento Didáctica de la Lengua y la Literatura Universidad de Granada. Correo: mssancho@ugr.es. 


\section{Introducción}

El desarrollo de los niños, así como el proceso de aprendizaje educativo, tienen mayor relevancia durante la etapa de la Educación Infantil. Esta es crucial para su crecimiento, ya que en ella se producen cambios que, seguramente, serán los más importantes que experimenta el ser humano a lo largo de su vida. Muñoz (2009, p. 25) indica muy acertadamente que "una educación de calidad no puede descuidar esta etapa, igualmente que no puede reducir sus finalidades al logro de determinados niveles de rendimiento académico de los niños". En este contexto, el lenguaje juega un papel fundamental. Es la forma que tienen las personas para comunicarse, para expresar sus pensamientos, sentimientos e ideas, siendo la lengua oral el principal vehículo de comunicación con los demás. De esta forma, la comunicación se convierte en el fundamento de la vida social y en la función primaria del lenguaje. En este sentido queremos destacar las palabras de Núñez (2011, p. 4) con quien coincidimos rotundamente:

La comunicación humana específica se basa en el uso del lenguaje, con respecto al cual se puede coincidir en que está hecho de señales arbitrarias producidas vocalmente y recibidas por vía auditiva y en que es sistemático, esto es, en que constituye un conjunto de elementos conectados entre sí que adquieren valor en virtud de sus relaciones con otros elementos del mismo sistema; por lo mismo, las lenguas son mecanismos de complejidad enorme a través de los cuales nos relacionamos con el mundo y lo transformamos en inteligible.

Desde un punto de vista funcional, el uso del lenguaje se concibe como una actividad humana compleja que garantiza las funciones básicas de comunicación y representación (Avedaño y Miretti, 2007). En esta misma línea debemos añadir que la lengua oral es la primera que aprendemos, por lo que estará presente en la mayoría de los aprendizajes que realizamos en la infancia. Siguiendo a Bigas (2008) podemos afirmar que es el principal instrumento que permite realizar un aprendizaje escolar satisfactorio, sobre el que se fundamentarán todos los conocimientos posteriores. Es por esto por lo que es especialmente relevante en esta etapa como herramienta principal de aprendizaje, de regulación de la conducta y de manifestación de vivencias, sentimientos, ideas, emociones, etc. Asimismo, otra de las funciones que desempeña consiste en potenciar el desarrollo del niño en el proceso de socialización, entendiendo este concepto como la causa por la cual el sujeto aprende y adquiere los elementos socioculturales del medio que lo rodea, moldeando sus actuaciones para adaptarse a la sociedad.

Este desarrollo lingüístico del niño depende en gran medida del medio social. Aprender a hablar -a usar la lengua- es una destreza social para la que el niño precisa modelos, necesita una guía adulta que le ayude a comprender lo que le rodea, que le ayude a expresarse, que lo escuche con interés y paciencia, que le ofrezca actividades interesantes en las que el lenguaje contribuya sustancialmente a organizar la acción y a asimilar lo vivido (Ruiz, 2002). Los docentes deben ser, además, modelos de variedad y corrección expresivas y fomentar en sus aulas interacciones ricas y abundantes creando un clima motivador y de participación que otorgue protagonismo a los alumnos y tenga en cuenta sus aportaciones.

En este sentido creemos que es necesario partir de la formación -los conocimientos disciplinares y didácticos- y las concepciones que poseen los docentes acerca de la enseñanza de la lengua oral en la Educación Infantil, ya que cualquier progreso en la educación tiene que prestar especial atención a la formación de los profesores. Por este motivo la pretensión de nuestra investigación es indagar en este tema, conocer cuáles son las concepciones y la formación del profesorado y, con todo ello, sentar las bases de 
lo que podría ser un futuro trabajo de investigación que, además, incluya una descripción de los conocimientos que realmente necesita el docente para conducir al niño a un desarrollo óptimo de la lengua oral, por medio de estrategias didácticas coherentes y sistemáticas.

Partiendo del propósito general de nuestra investigación, podemos evidenciar otros objetivos más específicos que recogemos a continuación:

a) Conocer las concepciones y los conocimientos que poseen los docentes de la Educación Infantil acerca de la importancia de la enseñanza de la lengua oral en esta etapa.

b) Analizar y describir cuáles son los métodos, técnicas y recursos utilizados por los docentes en esta etapa para la planificación y la práctica de la lengua oral.

c) Indagar en los elementos formativos que los docentes consideran como imprescindibles para alcanzar una práctica educativa de calidad.

d) Explorar y detectar qué capacidades pone en práctica el maestro de Educación Infantil cuando evalúa la destreza oral del alumnado.

\section{La lengua oral en la Educación Infantil y el papel del docente}

La Educación Infantil es una etapa prioritaria en cualquier sistema educativo moderno que va aproximadamente desde los cero hasta los seis años de edad. Su finalidad principal consiste en atender a los niños para garantizar su desarrollo físico, afectivo, social e intelectual, y es, sin duda, crucial para el proceso educativo y socializador.

Disponemos de la posibilidad de comunicarnos mediante diferentes sistemas y códigos, pero la len- gua oral es el medio más eficaz para la comprensión y producción de nuestros pensamientos, experiencias, sentimientos, deseos, opiniones, así como para la reflexión sobre nuestra propia identidad. La lengua es, por tanto, parte esencial del lenguaje. En palabras de O'Shanahan (1995, p. 47), la lengua "es un producto social, un código oral creado por cada sociedad y presente en la conciencia de sus individuos, que lo utilizan para comunicarse. Cada sociedad tiene su propia lengua, que es el sistema de signos y reglas aceptado por sus miembros y utilizado por éstos para comunicarse".

Y para que se produzca este acto de comunicación es imprescindible la existencia del entorno verbal que dé pie a su desarrollo y a la adquisición de un sistema lingüístico determinado.

En este sentido, el papel de la institución educativa $y$, por ende, del docente en cuanto al desarrollo y evolución del niño durante todo el proceso de su aprendizaje educativo es crucial en esta etapa. El rol del maestro será el de contribuir al correcto desarrollo y perfeccionamiento de la lengua oral en el niño, por lo que deberá disponer de una serie de conocimientos que le permitan desempeñar tales acciones, realizando diseños e intervenciones óptimas que contribuyan a enriquecer y a mejorar la multitud de aspectos comunicativos y lingüísticos implicados en la competencia oral.

Con los cambios introducidos en la actual legislación educativa, entre ellos la presencia de las competencias en el currículum, se le ha atribuido un mayor protagonismo a la lengua oral por este valor socializador que señalamos, sobre todo, en la etapa de la Educación Infantil. Este nuevo enfoque de la educación precisa un currículum basado en nuevas necesidades formativas y demanda un planteamiento profundo de los currículos y de los métodos de enseñanza y aprendizaje con un fin prioritario (Pérez y Zayas, 2007). Según el proyec- 
to DeSeCo (Definition and Selection of Competences) de la OCDE (2006) una competencia es más que conocimiento, en el caso de la competencia en comunicación lingüística hace referencia a la habilidad para usar la lengua, es decir, la puesta en práctica de las destrezas que adquirimos para establecer una interacción con el entorno garantizando nuestro desarrollo en la sociedad.

El trabajo didáctico sobre la lengua oral en el contexto escolar debe hacerse de forma planificada y estructurada, por lo que la práctica educativa de los docentes debe ser organizada para poder proporcionar así a los aprendices las herramientas y los conocimientos imprescindibles que aseguren una correcta adquisición de su competencia oral. Como pauta didáctica general en esta etapa, es más adecuado empezar con textos orales sencillos (trabalenguas, retahílas fáciles o juegos) para ir avanzando hacia propuestas más complejas, por lo que se requiere una planificación graduada y meditada sobre los textos seleccionados y las actividades propuestas (Núñez y Santamarina, 2010). Los maestros de Educación Infantil deben contar con un amplio y adecuado repertorio de actividades sobre tipos de textos diversos y hacer uso de prácticas sistematizadas en las que el niño participe y pueda expresarse en un ambiente de libertad, seguridad afectiva y emocional, así como recibir retroalimentación y corrección, lo cual incrementará sus posibilidades de progreso en el logro de la competencia lingüística.

\section{Conocimiento, concepciones y saberes de los docentes}

Durante la acción educativa, tanto los profesores ya formados como los profesores en formación adquieren una serie de concepciones sobre el aprendizaje y la enseñanza que guían su práctica a lo largo de su ejercicio profesional. Este conocimiento base posee un carácter implícito y difiere en algunas ocasiones de las concepciones explícitas que manifiestan.

Como acertadamente evidencia Rodrigo (1997), las teorías implícitas proporcionan el marco conceptual, epistemológico y ontológico a partir del cual el sujeto elabora las teorías de dominio, de manera que restringen tanto la selección de la información procesada como las relaciones establecidas entre los elementos de esa información.

En palabras de Pozo y otros (2006), entender las concepciones como teorías implícitas comporta la idea de que son representaciones complejas y muestran diferentes aspectos, según la mirada y el tipo de indagación que se realice. Estos autores añaden que es necesario realizar un análisis del conjunto de producciones e indagaciones que apunten a una misma dirección para atribuir una determinada teoría sobre el aprendizaje y la enseñanza a los docentes implicados.

Este enfoque, las concepciones del aprendizaje como teorías implícitas y el pensamiento del profesor constituyen el marco teórico que mejor representa nuestro planteamiento en este trabajo. Al igual que sucede con las concepciones de los docentes, los saberes que sustentan la labor educativa de los maestros se encuentran, por norma general, implícitos en las prácticas educativas específicas a lo largo del proceso de enseñanza y aprendizaje.

Acerca de los saberes, Mercado (1991, p. 60) señala que "la noción de saber remite al conocimiento sobre la realidad que utilizamos de un modo efectivo en la vida cotidiana del modo más heterogéneo".

A lo largo de su ejercicio profesional, el docente concreta y establece los saberes que conforman su práctica diaria. Estos saberes incluyen información relativa a la enseñanza que le ha sido significativa durante su formación académica y sirven para resolver los problemas que surgen a lo largo del ejercicio profesional, por lo que dan sentido a las 
situaciones de trabajo que le son propias. Vilanova, García y Señorino (2007) señalan que los resultados de las investigaciones sobre concepciones y creencias de los docentes, han mostrado que tanto los profesores ya formados como los alumnos de los profesorados, conciben y se forman representaciones del aprendizaje y la enseñanza de modo intuitivo.

La profesión de enseñar exige la puesta en práctica de estos saberes por parte del docente y ellos le servirán para estructurar y orientar su actividad profesional. De esta forma, podemos encontrar saberes que se relacionan con la capacidad organizativa del docente o, en palabras de Mercado (1991), con saber organizar al grupo en un trabajo común. Otros saberes remiten a los recursos que utiliza para captar el interés de los alumnos en el trabajo o a los relacionados con la actividad individual de los alumnos. Asimismo, la observación, la descripción y el análisis de los saberes docentes son posibles a través de una estrategia de investigación pertinente a ese objeto de conocimiento, lo que implica una conceptualización que presuponga su existencia.

En el caso de la enseñanza de la lengua, investigadores de la talla de Van Lier (1995) han apuntado que es preciso aumentar el grado de conciencia de los docentes respecto de su intervención en la práctica de la oralidad en el aula para que esta se dirija a la mejora de las habilidades de comunicación de los niños. Es por ello que son necesarias más investigaciones de diferente envergadura que analicen la naturaleza de las concepciones y su relación con la práctica educativa, para así emplear estos conocimientos en la creación de modelos de formación que supongan un cambio conceptual, atendiendo a las demandas actuales de nuestra sociedad.

\section{Metodología}

La investigación llevada a cabo se enmarca en la metodología cualitativa, concretamente en el enfoque del estudio de caso (Walker, 1983) de tipo colectivo, que contiene una serie de datos descriptivos que, extraídos de transcripciones de entrevistas como fuente de evidencias, han sido codificados, categorizados y ordenados de forma sistemática para que se muestren congruentes y ayuden a determinar las claves del pensamiento y las concepciones de los sujetos entrevistados. Asimismo, también posee ciertas características que la encuadran dentro del método clínico; una de ellas es la utilización de la entrevista clínica como método de recogida de los datos. Con este tipo de investigación no buscamos el caso representativo sino de estar atentos a lo que se puede aprender del estudio del caso concreto o del grupo de casos. Coincidimos con Stake (2005) en que el equilibrio y la variedad son importantes, pero la oportunidad para aprender resulta clave y esencial.

El método empleado en esta investigación, el método clínico, se caracteriza por su extraordinaria flexibilidad, lo que permite ajustarse a las conductas del sujeto y así poder encontrar el sentido de lo que va haciendo y diciendo. Asimismo, es un excelente instrumento para descubrir la construcción de las representaciones que poseen las personas acerca de la realidad (Delval, 2001).

La realización de un estudio piloto en este tipo de investigaciones resulta de extrema importancia porque posibilita probar el procedimiento de investigación antes de emprender trabajos de más alcance ${ }^{1}$. En este caso, el estudio preliminar se ha realizado con un número reducido de sujetos, pues buscamos

1 Como hemos señalado previamente, nuestra intención será la de continuar con la investigación aquí iniciada por tanto los objetivos serán notablemente ampliados a medida que la investigación avance y el número de sujetos que intervengan en la muestra sea más amplio, es por ello por lo que podemos considerar esta investigación como un estudio piloto, ya que nos permitirá variar las ideas iniciales. 
ante todo aquilatar el instrumento, la entrevista, así como abordar aspectos no previstos inicialmente, pero que han surgido de las respuestas de las dos entrevistadas. El estudio piloto también nos ha permitido descubrir si nuestras preguntas resultan comprensibles para los sujetos y si hemos obtenido respuestas ricas y valiosas o si han sido puramente estereotipadas.

El instrumento seleccionado para acceder a las concepciones de los docentes ha sido la entrevista clínica. En este sentido, la entrevista es posiblemente el instrumento más utilizado en la investigación cualitativa y, más aún, en los estudios de caso. Pades (2006) detalla sus principales características, las cuales son comunes a toda entrevista independientemente del enfoque que la defina: su comunicación verbal y no verbal, cierto grado de estructuración, una finalidad específica, su situación asimétrica, el proceso bidireccional y la adopción de roles específicos por ambas partes. López (2007) apunta que en la entrevista clínica no buscamos un dato, sino información, ya que esta, vista como conjunto de datos situados en un contexto, resulta más enriquecedora.

De acuerdo con lo expuesto, la investigación se ha desarrollado conforme a las siguientes fases, las cuales se explican aquí muy brevemente. Estas fases corresponden a los pasos principales del método clínico.

1. Seleccionar el problema y definirlo con precisión. Analizar cuáles son las concepciones y conocimientos de los docentes acerca de la enseñanza de la lengua oral en la etapa de la Educación Infantil.

2. Examinar los antecedentes del trabajo. Se ha realizado una revisión bibliográfica para establecer la fundamentación teórica de nuestro trabajo, así como para encontrar otros referentes que traten el mismo o un tema similar de investigación.
3. Planificación de la recogida de datos. Debido al carácter de la investigación, y una vez fijados el problema y los objetivos que pretendíamos conseguir, nos decantamos por utilizar la entrevista clínica semiestructurada para la recogida de los datos. Se ha utilizado una grabadora digital de sonidos para registrar las entrevistas y así poder transcribirlas para su posterior análisis.

4. Selección de los participantes. Se han escogido dos maestras en ejercicio de Educación Infantil, una de ellas con poca experiencia profesional, por su edad, y la otra con amplia experiencia, pues le quedan pocos años para jubilarse. La elección se ha realizado de acuerdo con dos criterios: tener experiencia docente y estar ejerciendo la docencia actualmente en la etapa de Educación Infantil.

5. Recogida de datos. Se adoptó, al respecto, como modelo la entrevista clínica semiestructurada, sin impedir por ello las manifestaciones libres que los sujetos quisieran formular ante el entrevistador. Para la realización de las dos entrevistas se ha confeccionado un modelo de entrevista clínica procedente de una previa elaborada por la Dra. María Pilar Núñez Delgado (Universidad de Granada) acerca del pensamiento y las concepciones del profesor sobre la didáctica de la lengua oral, a la que hemos adaptado las cuestiones para abordar específicamente la enseñanza de la lengua oral en la Educación Infantil. La entrevista puede dividirse en dos secciones: 1. biografía personal del entrevistado; 2. preguntas acerca de la enseñanza de la lengua oral en la Educación Infantil.

Análisis de la información. La información obtenida ha sido sometida al oportuno proceso de reducción de evidencias y se ha continuado con la realización de un análisis de contenido. El análisis del discurso se ha realizado a través de la descripción y reflexión de la información obteni- 
da por parte de las entrevistadas. Este proceso se apoya en la distribución del contenido en categorías y metacategorías, obtenidas de forma inductiva, y organizadas con la ayuda del programa de análisis de datos Nvivo-v.9. De esta forma, una vez que se hubieron transcrito literalmente las entrevistas, se procedió a la división de los textos en unidades de contenido (Bardin, 1986), a las que se aplicaron los códigos.

6. En total, se han establecido y aplicado 37 códigos, agrupados posteriormente en siete (7) metacategorías: formación, lengua oral, planificación, elementos curriculares, alumnado de Educación Infantil, enseñanza de la lengua oral y evaluación de la lengua oral.

Las entrevistas se realizaron en momentos y situaciones totalmente diferentes. La primera entrevista, por cercanía personal, se realizó en la casa de la entrevistadora donde se dispuso del tiempo necesario para llevarla a cabo. No se produjeron interrupciones y el ambiente fue relajado y adecuado para lograr nuestro propósito. El tiempo de duración de la entrevista fue de 46 minutos y 61 segundos. La segunda entrevista se realizó en condiciones más adversas, pues el espacio físico fue el centro de trabajo de la entrevistada, concretamente, la sala de profesores, y aunque se dispuso de tiempo suficiente para llevar a cabo la entrevista, se produjo alguna que otra interrupción, contando con que la entrevistada tenía que dar clase, lo cual se constata en la duración de esta segunda entrevista: 22 minutos y 36 segundos.

\section{Resultados y su discusión}

Una vez codificada y analizada la información a través de las categorías establecidas, obtenidas inductivamente -como ya se ha señalado-, se procedió a la presentación de los resultados obtenidos mediante una descripción del discurso, destacando los aspec- tos más significativos relativos a cada una de las metacategorías y categorías fijadas.

Los siguientes puntos recogen los principales resultados de las entrevistadas. Ambas son maestras de Educación Infantil en ejercicio en Andalucía (España).

\section{Resultados: primera entrevistada}

- La Formación Profesional (FP) le ha proporcionado más elementos teóricos y metodológicos para enseñar lengua oral que la universidad. Asimismo, resalta en numerosas ocasiones la importancia de la formación continua del profesorado, hecho que debería de promover la propia escuela.

- La concepción que posee la entrevistada acerca de la enseñanza de la lengua oral en esta etapa repercute de forma directa en la enseñanza de la misma en la escuela. La lengua oral, a su entender, implica la actuación de diversos aspectos verbales y no verbales.

- Los fines de la enseñanza de la lengua oral en esta etapa educativa dependen, a su juicio, del ciclo; asimismo, para la entrevistada el objetivo primordial de la lengua oral en Educación Infantil debe ser el de conseguir que el niño exprese sus deseos, sus motivaciones y sus necesidades y que pueda desenvolverse en la vida diaria.

- En relación con las estrategias de enseñanza que lleva a la práctica, señala que la motivación y la innovación son dos requisitos indispensables; asimismo, los recursos empleados proceden, en su mayoría, de los que le proporciona el método.

- Con respecto al tiempo empleado en la enseñanza de la lengua oral, apunta que esta "siempre se trabaja" al igual que la literatura, aunque apunta que es en la asamblea donde realmente se trata la lengua oral en profundidad. 
- Los tipos de actividades que emplea para la enseñar lengua oral son, sobre todo, las canciones, poesías, bailes y representaciones teatrales.

- Sobre la asamblea, reconoce que es el momento en el que trabaja, a su modo de ver, de forma más clara la lengua oral con sus alumnos.

- La entrevistada hace numerosas referencias a la importancia que para ella tiene la escucha. "Escuchar a los alumnos para que ellos aprendan que tienen que escuchar a los demás”.

- Las rutinas y las normas son conceptos que considera imprescindibles en la Educación Infantil.

- En lo referente a la coordinación del profesorado de Infantil con el de Primaria en lo relativo a la lengua oral, la entrevistada señala que normalmente esto nunca se produce, ni se continua el método iniciado en la etapa de Educación Infantil, hecho que, a su modo de ver, perjudica, sin lugar a dudas, a los alumnos.

\section{Resultados: segunda entrevistada}

- Hace especial hincapié en la necesidad de formación después de la enseñanza universitaria, donde apunta que no se aprende lo suficiente; asimismo, atribuye un papel clave a los contenidos prácticos.

- La entrevistada señala que, a su modo de ver, el concepto oralidad hace referencia a la "capacidad de comunicar las cosas", y apunta que la finalidad de la enseñanza de la lengua oral en esta etapa educativa debe ser la de proporcionar al alumno la posibilidad de expresarse, de comunicarse.
- Acerca de los objetivos y contenidos relacionados con la lengua oral que componen el currículum de la etapa, considera que son suficientes y que el currículum es bastante completo en este sentido. Indica que conoce a fondo lo regulado en el currículum de la etapa.

- Destaca que las habilidades de lengua oral se trabajan bastante en la Educación Infantil. En su caso, tal y como nos indica, da mayor importancia a la expresión del alumno y explica que trata de corregirlo de forma directa, para que el niño se dé cuenta de su error.

- En cuanto a las características del alumno de Educación Infantil, la entrevistada expone que hay que motivarlo para que se implique en las actividades.

- Las actividades relacionadas con la lengua oral que trabaja en clase son, sobre todo, las poesías, las canciones, las representaciones teatrales y la asamblea.

- Los textos orales que más trabaja son las poesías, las canciones y los cuentos.

- El papel de la asamblea para la entrevistada es crucial en el desarrollo de la lengua oral en el alumno.

- El uso de las rutinas y de las normas es más evidente que el de los rincones ${ }^{2}$, los cuales apenas introduce en su discurso.

- Acerca de la coordinación con el profesorado de Educación Primaria, concluye que es una difícil tarea, aunque en el centro en el que se encuentra actualmente siempre se procura que exista una colaboración mutua.

2 Los rincones de trabajo nos permiten gestionar y organizar el aula en grupos reducidos de alumnos donde cada niño lleva a cabo una tarea concreta y diferente. De esta forma aprenden a trabajar en equipo poniendo en práctica los conocimientos que han adquirido a la vez que aprenden nuevos contenidos y desarrollan su autonomía. 


\section{Conclusiones}

Una vez finalizado el proceso de investigación, se pueden evidenciar las principales conclusiones a las que se ha podido llegar con este estudio de casos en relación con cada una de las metacategorías consideradas en el mismo:

\section{Sobre la formación recibida}

Las maestras entrevistadas tienen una pronunciada diferencia de edad: 29 y 58; es por ello que su formación previa es significativamente desigual, aunque en ambos casos se puede constatar que, aparte de Magisterio de Educación Infantil, poseen otros estudios. Las dos coinciden en la necesidad de ampliar su formación lingüística, la cual, como indican, no se aprende realmente en la universidad. Asimismo, señalan que es importante continuar realizando cursos prácticos sobre la enseñanza.

Acerca de las personas que influyeron en la elección de su carrera, destacan algunos familiares y docentes, aunque reconocen que desde la niñez se sienten motivadas a estudiar Magisterio, alegando en el caso de la segunda entrevistada su gusto por la enseñan$\mathrm{za}$, aspecto que consideramos fundamental para dedicarse a la profesión de docente.

\section{Sobre la lengua oral}

Las entrevistadas proporcionan una definición de la oralidad y de la lengua oral derivada, sobre todo, de su práctica educativa y con escaso aparato teórico y didáctico como soporte. Ambas señalan la importancia de la enseñanza de la lengua oral para que el niño pueda comunicarse y expresar sus sentimientos, ideas, emociones y un largo etcétera, sin embargo, debemos destacar que las definiciones son, sobre todo en el caso de la segunda entrevistada, breves, quizá debido a la formación lingüística que las en- trevistadas señalan no haber recibido correctamente a lo largo de sus estudios universitarios, aunque, evidentemente, no debemos olvidar que una de las limitaciones que posee la entrevista es el olvido en que pueden caer los entrevistados, originado por la tensión generada al sentirse examinados, más aún durante los primeros minutos.

Asimismo, no tratan en un primer momento el tema de las habilidades lingüísticas orales hasta que la entrevistadora les pregunta directamente. A este respecto, las entrevistadas no atribuyen la misma importancia a las habilidades; de esta forma, la primera entrevistada indica que suele dar mayor prioridad a la comprensión oral, la escucha, y la segunda entrevistada otorga más importancia a la expresión oral, es decir, al habla.

Como hemos podido comprobar en la descripción de los resultados, las entrevistadas apuntan en numerosas ocasiones la importancia de la lengua oral en la etapa de la Educación Infantil, indicando ambas que en sus clases procuran llevar a cabo actividades que la trabajen y la desarrollen en el alumno; sin embargo, y tal y como hemos apuntado en la fundamentación teórica, la mayoría de las actividades empleadas no son suficientes para lograr tal propósito. Para trabajar, por ejemplo, las habilidades lingüísticas orales son necesarias diversas estrategias que favorezcan la comprensión y expresión del niño, y en este sentido, las utilizadas por estas maestras son escasas y poco variadas, quedándose anquilosadas en unas que no profundizan lo suficiente ciertos aspectos fundamentales de la lengua oral, tales como la intensidad de la voz, la respiración, las pautas del habla, el tiempo del habla y otros elementos relacionados con la expresión y la comprensión orales.

A lo largo del discurso las entrevistadas exponen los tipos de textos que trabajan en sus respectivas clases, y al respecto debemos evidenciar que sue- 
len utilizar textos orales muy similares, tales como los narrativos (cuentos o fábulas) y los literarios (poesías, canciones y textos teatrales), descuidando otros muchos que contribuyen a desarrollar la lengua oral $-y$ la competencia comunicativa en su conjunto- en el alumno: nos referimos a los textos argumentativos, descriptivos, dialogados o instructivos, entre otros. Asimismo, el uso de retahílas, juegos de palabras, adivinanzas, es decir, la literatura de tradición oral, se convierte en una herramienta efectiva para mejorar el vocabulario y alcanzar ciertos objetivos y destrezas lingüísticas, entre otros diversos fines.

Ambas entrevistadas vuelven a coincidir en la atribución de un papel principal a la asamblea como actividad que desarrolla y trabaja las destrezas orales del alumno. Consideran que es una parte fundamental en la Educación Infantil, pero no observamos que se realice una planificación previa estructurada o que las actividades que se llevan a cabo en ella, así como las estrategias metodológicas empleadas, sean claras ni estructuradas con arreglo a ningún criterio didáctico. De hecho, ambas mencionan que es una actividad algo rutinaria porque siempre se desarrolla de modo muy similar. En este sentido, no podemos olvidar que la asamblea es un medio muy enriquecedor para contribuir a este desarrollo de la lengua oral en el niño, pero necesita actividades, estrategias y mecanismos que garanticen tal propósito; es decir, no basta con hablar con el niño en círculo, hay que aplicar mecanismos de apoyo para enriquecer y corregir su lenguaje en todos los aspectos que integran la competencia oral: coherencia, cohesión, corrección, adecuación, etcétera.

El uso de rutinas y normas en clase es otro de los aspectos clave en esta etapa. Sobre este tema las entrevistadas señalan con gran acierto que en la Educación Infantil es fundamental enseñar a los alumnos qué son las rutinas y las normas y cuándo hay que utilizarlas, todo ello a través de la práctica diaria. En el caso de las normas, pueden llevarse a cabo actividades que enseñen de forma indirecta la importancia de estas para desenvolverse de forma adecuada en la sociedad, a través, por ejemplo, de juegos. En este sentido, las entrevistadas no señalan si emplean alguna práctica concreta para fomentar el uso de normas o rutinas, sino que añaden que las suelen trabajar de forma constante en el aula y a través de la asamblea; tampoco hacen comentarios específicos sobre el componente verbal y de aprendizaje de modelos textuales que suponen las rutinas y las normas en el aula, de donde cabe concluir que, al no ser conscientes de ello, no rentabilizan didácticamente estas situaciones de interacción.

\section{Relacionadas con la planificación}

La planificación de la enseñanza es, sin duda, una tarea capital en el proceso educativo. Una planificación adecuada proporciona a los docentes diferentes estrategias y recursos para mejorar la práctica en el aula. Es cierto que la enseñanza es un proceso dinámico y que muchas de sus variantes escapan al control y, por tanto, a la planificación. Es por ello que se debe renovar y cambiar la planificación para que esta sirva de apoyo durante la práctica docente. En el caso de las entrevistadas, no especifican de forma clara si planifican con tiempo y si renuevan la planificación de la lengua oral en el aula, a pesar de que a lo largo de la entrevista se procura indagar en este tema, con lo cual puede deducirse que el tiempo empleado en la planificación no es muy extenso y que esta no se plasma en documentos de ningún tipo, más allá de los requeridos por la administración educativa a comienzos de cada curso.

\section{Respecto de la evaluación de la lengua oral}

La evaluación educativa demanda un uso adecuado de diversos métodos y estrategias que posibiliten el 
acceso al conocimiento acerca de lo que ocurre a lo largo y al final de cada secuencia didáctica, observando aquello que se pretende conseguir y lo que realmente se enseña en el aula.

En este sentido, las entrevistadas no recogen este aspecto en el sistema que emplean para evaluar la lengua oral en sus clases; la primera de ellas detalla que lleva a cabo una "evaluación final" a través de un juego con el que puede comprobar si los alumnos han conseguido los objetivos prefijados, y la segunda utiliza la observación directa como principal instrumento de evaluación. Estos métodos son escasos, si no se ponen en práctica junto con otros que incluyan la revisión de las estrategias y recursos utilizados por los docentes, además de que son insuficientes para evaluar la lengua oral en el alumno. Por ejemplo, y como ya hemos comentado, la evaluación diagnóstica puede ser de gran ayuda para analizar la capacidad expresiva y comprensiva del niño y así trabajar en su potencial lingüístico concreto.

\section{A modo de síntesis final}

Una vez realizado el estudio exhaustivo de las entrevistas, podemos evidenciar las siguientes conclusiones:

- Las concepciones y conocimientos acerca de la competencia oral y su didáctica por parte de los maestros de Educación Infantil se ajustan más a modelos tradicionales de enseñanza de la lengua que a los requerimientos de los actuales enfoques comunicativos y funcionales exigidos por el currículum vigente basado en competencias.

- El tiempo destinado por los docentes de Educación Infantil a la planificación y a la práctica de la lengua oral es escaso en relación con las necesidades de los niños en esta etapa.
- Existen disparidades en las maneras de entender, planificar y llevar a la práctica la enseñanza de la lengua oral en la Educación Infantil entre docentes de diferentes edades y trayectorias profesionales.

- La formación de los docentes en relación con las competencias básicas, y específicamente en la competencia en comunicación lingüística y la forma de lograr su desarrollo en el alumnado, es algo anticuada e insuficiente.

- La evaluación del desarrollo de las capacidades orales del alumnado de Educación Infantil carece de sistematicidad y no utiliza instrumentos y técnicas rigurosas.

Siguiendo a Sánchez (2009) a lo largo de la práctica educativa, los docentes no asumen conscientemente la diversidad de aspectos en los que es posible intervenir para que el espacio del aula sea favorable y enriquecedor respecto del desarrollo de la competencia comunicativa en la Educación Infantil según la normativa vigente. En fin, queremos hacer un especial hincapié en que la investigación que conforma este trabajo pretende, como consecuencia de las evidencias encontradas y expresadas en los puntos anteriores, poner de manifiesto que hay una clara necesidad de mejorar y actualizar tanto la formación inicial como la formación permanente del profesorado de Educación Infantil, desde un punto de vista científico y didáctico en lo relativo a la competencia oral y al modo de lograr su desarrollo en el alumnado de esta etapa.

\section{Reconocimientos}

Este artículo presenta análisis de las concepciones del profesorado en Educación Infantil sobre la enseñanza de la lengua oral. 


\section{Bibliografía}

Avedaño, F. y Miretti, M. L. (2007). El desarrollo de la lengua oral en el aula. Sevilla: MAD.

Bigas, M. (2008). El lenguaje oral en la escuela infantil. Glosas didácticas. Revista electrónica internacional, 17. Recuperado de:

http://www.um.es/glosasdidacticas/numeros/ GD17/03.pdf

Bardin, L. (1986). El análisis de contenido. Madrid: Akal.

Delval, J. (2001). Descubrir el pensamiento de los niños. Introducción a la práctica del método clínico. Barcelona: Paidós.

López, M. (2007). Comunicación médico paciente en entrevista clínica. Revista El Médico, 4.

Mercado, R. (1991). Los saberes docentes en el trabajo cotidiano de los maestros. Infancia y Aprendizaje, 55, 59-72. Recuperado de dialnet.unirioja.es/servlet/dcfichero_ articulo? codigo $=48375$

Muñoz, A. (coord.) (2009). El desarrollo de las competencias básicas en Educación Infantil. Sevilla: MAD.

Núñez, M. P. (2011). Didáctica de las habilidades lingüísticas en la educación inicial. Madrid: ITEM Multimedia.

Núñez, M. P. y M. Santamarina (2010). Literatura de tradición oral y educación literaria en la primera infancia. Alhucema. Revista internacional de teatro y literatura, 24, 220-233.

OCDE (2006). Definición y selección de competencias clave. Resumen ejecutivo. Recuperado de http://www.deseco.admin.ch/bfs/deseco/en/ index/03/02.parsys

O'Shanahan (1995). Enseñanza del lenguaje oral y las teorías implícitas del profesorado. (Tesis doctoral) Universidad de La Laguna. Recuperado de

ftp://tesis.bbtk.ull.es/ccssyhum/cs27.pdf

Pades, A. (2006). La entrevista clínica: herramienta para cuidar. Ágora de enfermería, 5, 1-8. Recuperado de

http://www.agoradenfermeria.eu/CAST/ num005/inferm.html

Pérez, P. y Zayas, F. (2007). Competencia en comunicación lingüística. Madrid: Alianza.

Pozo, J. I. (coord.) (2006). Nuevas formas de pensar la enseñanza y el aprendizaje. Las concepciones de profesores y alumnos. Barcelona: Graó.

Rodrigo, M. J. (1997). Del escenario socio-cultural al constructivismo episódico: un viaje al conocimiento escolar de la mano de las teorías implícitas. En M. J. Rodrigo y J. Arnay (eds.). La construcción del conocimiento escolar. Barcelona: Paidós.

Ruiz Bikandi, U. (2002). La construcción de la lengua oral. Aula de Infantil, 10, 6-11.

Sánchez, S. (2009). La práctica de la asamblea en clase en la educación infantil y la formación de maestros en didáctica de la lengua oral. Lenguaje y textos, 30, 195-206.

Stake, R. (2005). Investigación con estudios de caso. Madrid: Morata SRL.

Van Lier, L. (1995). Lingüística educativa: una introducción para enseñantes de lenguas. Signos: Teoría y práctica de la Educación, 14, 20-29. 
Vilanova, S; M. García y O. Señorino (2007). Concepciones acerca del aprendizaje: diseño y validación de un cuestionario para profesores en formación. Revista electrónica de investigación educativa, 9(2). Recuperado de http://redie. uabc.mx/vol9no2/contenido-vilanova.html
Walker, R. (1983). La realización de estudios de casos en educación. Ética, teoría y procedimientos. En W. B. Dockrell y D. Hamilton (comps.). Nuevas reflexiones sobre la investigación educativa. Madrid: Narcea. 